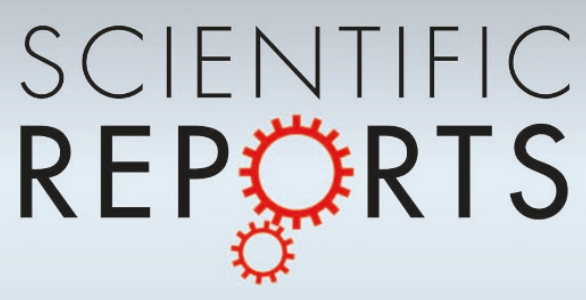

OPEN

SUBJECT AREAS:

NANOPARTICLES

SYNTHESIS AND PROCESSING

Received

25 January 2013

Accepted

13 August 2013

Published

28 August 2013

Correspondence and requests for materials should be addressed to M.-W.M. (mwmoon@ kist.re.kr)

\section{Porous Carbon Nanoparticle Networks with Tunable Absorbability}

\author{
Wei Dai ${ }^{1}$, Seong Jin Kim ${ }^{1}, 2$, Won-Kyung Seong' , Sang Hoon Kim ${ }^{1}$, Kwang-Ryeol Lee' ${ }^{1}$ Ho-Young Kim ${ }^{2}$ \\ \& Myoung-Woon Moon'
}

'Institute for Multi-disciplinary Convergence of Matter, Korea Institute of Science and Technology, Seoul 130-650, Republic of
Korea, ${ }^{2}$ School of Mechanical and Aerospace Engineering, Seoul National University, Seoul 151-744, Republic of Korea.

Porous carbon materials with high specific surface areas and superhydrophobicity have attracted much research interest due to their potential application in the areas of water filtration, water/oil separation, and oil-spill cleanup. Most reported superhydrophobic porous carbon materials are fabricated by complex processes involving the use of catalysts and high temperatures but with low throughput. Here, we present a facile single-step method for fabricating porous carbon nanoparticle (CNP) networks with selective absorbability for water and oils via the glow discharge of hydrocarbon plasma without a catalyst at room temperature. Porous CNP networks were grown by the continuous deposition of CNPs at a relatively high deposition pressure. By varying the fluorine content, the porous CNP networks exhibited tunable repellence against liquids with various degrees of surface tension. These porous CNP networks could be applied for the separation of not only water/oil mixtures but also mixtures of liquids with different surface tension levels.

uperhydrophobic materials with a static water contact angle (CA) exceeding $150^{\circ}$ and low contact angle hysteresis (CAH) of less than $10^{\circ}$ have attracted significant attention owing to their unique water-repellent and self-cleaning properties and due to their potential and practical applications, which range from biotechnology and environmental and water technology to self-cleaning commercial materials ${ }^{1-4}$. The superhydrophobicity of surfaces is generally based on a combination of chemical composition and topography. A principal characteristic of superhydrophobic surfaces is multiscale roughness on the micro- and nano-scale combined with a coating material with low surface energy, on which air can be trapped between grooves and repel liquid droplets ${ }^{2,3}$. Recently, carbon materials such as amorphous carbon coatings, graphene foams or sponges, carbon nanotube forests, carbon fibers, carbon nanowalls and porous carbon materials have been considered as suitable candidates for superhydrophobic surfaces due to their low surface energy and tunable topological nanostructures ${ }^{5-12}$. In particular, due to their high surface area and large pore volume, porous carbon materials or structures have been used extensively as electrode materials for batteries and super-capacitors, as sorbents for separation processes and gas storage, and as supports for many important catalytic processes ${ }^{13-17}$. The combination of superhydrophobicity and porosity may broaden the potential application range of porous carbon materials to include, for instance, applications as water filtration or water/oil separation, gas diffusion media for fuel cells, and nonwetting liquid transfer materials ${ }^{18-21}$.

Numerous techniques have been developed to synthesize porous carbon materials, including chemical or physical activation, the wet-chemical route, the template replica process, and chemical vapor deposition $(\mathrm{CVD})^{22-25}$. Among these methods, CVD is one of the most common methods used to deposit carbon materials such as carbon nanotubes, carbon nanowalls, and other carbon-based nanostructured materials ${ }^{26-28}$. However, high-temperature processing and catalysis are usually required to synthesize most carbon-based nanostructured materials, which would limit the application of carbon nanostructures on materials with low melting points, including biomaterials such as polymers or paper. Recently, Banerjee et al. ${ }^{5}$ successfully prepared superhydrophobic amorphous carbon films with various nanostructured surfaces using plasma-enhanced CVD (PECVD), indicating that the deposition pressure was one of the most important and easily tuned parameters in controlling the growth morphology of the carbon materials deposited using PECVD. However, because the synthesis of most superhydrophobic carbon materials is based on the ability to control surface features or properties, such materials may not be feasible for use in applications that require liquid or gas absorption.

Here, we report a facile single-step method for fabricating porous carbon nanoparticle (CNP) networks with tunable wettability and absorbability. Carbon materials were prepared by glow discharge deposition at pressures of $100 \sim 500$ mTorr using $\mathrm{C}_{2} \mathrm{H}_{2}$ and a gas mixture of $\mathrm{C}_{2} \mathrm{H}_{2}$ and $\mathrm{CF}_{4}$ as the carbon precursors. Porous $\mathrm{CNP}$ 


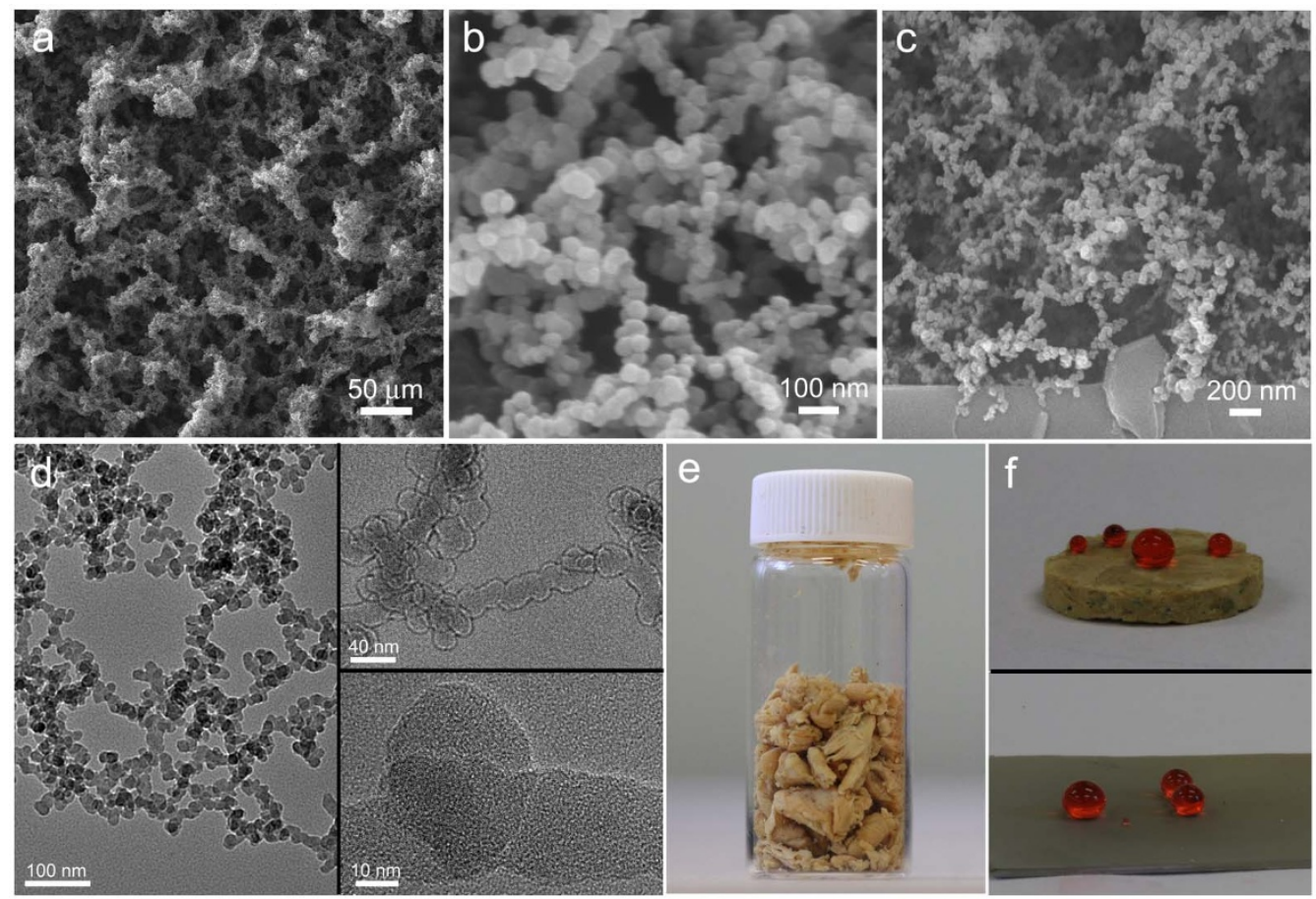

Figure 1 Structure and wettability of the porous CNP network deposited at $\mathbf{5 0 0}$ mTorr. (a) SEM images of the CNP network materials; (b) detailed view of the SEM image; (c) cross-sectional view of the porous CNP network; (d) corresponding TEM image of the porous CNP network; (e) optical image showing a collection of porous CNP materials deposited for $30 \mathrm{~min}$ in a vial measuring $2 \mathrm{~cm}$ in diameter; (f) water droplets placed on the porous CNP network coated onto the Si substrate (lower panel) and on the collected porous CNP networks upper, as formed into discs that retain the superhydrophobicity of the networks.

network materials were acquired at high deposition pressures above $200 \mathrm{~m}$ Torr at a low temperature $\left(<50^{\circ} \mathrm{C}\right)$, facilitating the coating of these materials onto other materials such as paper, polymers and metals. Furthermore, the porous CNP network materials with various fluorine contents showed the selective repellency and absorption of liquids with different surface tensions, making it possible for them to be used as benign materials in various applications, including the areas of water filtration, liquid separation or oil-spill (or organics) cleanup.

\section{Results}

SEM images of the porous carbon materials (Fig. 1a-c) show loose, fractal-like porous networks composed of CNPs with diameters of approximately $30 \sim 50 \mathrm{~nm}$. TEM images (Fig. 1d) show that these CNPs agglomerate to form particle chains and networks. In addition, high-resolution TEM also indicates that the CNPs display a typical amorphous structure without any lattice fringes. The formation of the amorphous structures may be attributed to the catalysis-free deposition process and the low deposition temperature ${ }^{29,30}$. The porous CNP network material was characterized by its nitrogen adsorption isotherm, which revealed a specific surface area of $148 \mathrm{~m}^{2} / \mathrm{g}$ (fitting of the Brunauer-Emmett-Teller, BET equation) (shown in Fig. S1). The pore size distribution of the porous CNP network calculated from nitrogen desorption branches shows a typical hierarchical pore size distribution, with the highest peak centered at $34 \mathrm{~nm}$ and several lower peaks centered at $15 \mathrm{~nm}$ and $5 \mathrm{~nm}$; this indicates that the majority of the pores could be considered mesopores. Due to the porous nature of CNP networks, the deposition yield of the carbon materials was measured to be $30 \mu \mathrm{m} / \mathrm{min}$, as indicated by the optical image in Fig. 1e, which shows a collection of porous CNP materials deposited at $500 \mathrm{mTorr}$ for $30 \mathrm{~min}$ in a vial measuring $2 \mathrm{~cm}$ in diameter. This growth rate was calculated to be 410 times faster than that of a dense and flat carbon coating deposited at $73 \mathrm{~nm} / \mathrm{min}$ at $100 \mathrm{mTorr}$. It is also interesting that the porous carbon material presented superhydrophobic properties, with a CA of approximately $150^{\circ}$ on an as-deposited film (lower image of Fig. 1f), which was maintained even on a porous CNP disc produced by mechanical compaction using a load of approximately $10 \mathrm{~N}$ (the upper image of Fig. 1f). The smooth carbon coatings deposited at a relatively low pressure of 100 mTorr showed an approximate CA of only $60^{\circ}$ (see Fig. S2).

The growth morphology of the as-deposited carbon materials was significantly correlated with the deposition pressure. Figure 2a shows the relatively smooth surfaces which were observed in materials deposited at 100 and 200 mTorr, while porous CNP structures suddenly appeared above $300 \mathrm{mTorr}$. Fig. $2 \mathrm{~b}$ presents the Raman spectra of the as-deposited carbon coatings as a function of the deposition pressure. All of the Raman spectra exhibit a broad asymmetric Raman scattering curve in the range of $1000 \sim 1700 \mathrm{~cm}^{-1}$ due to the presence of $\mathrm{sp}^{2}$ carbon bonds, representing the characteristic peak of amorphous carbon $^{31}$. This indicates that the as-deposited carbon materials, both the flat carbon and the porous CNP networks, are predominantly amorphous, in agreement with the TEM analysis. Normally, asymmetric Raman peaks can be fitted with two peaks: the G-peak around $1580 \mathrm{~cm}^{-1}$ and the D-peak around $1360 \mathrm{~cm}^{-1}$, which correspond to the $\mathrm{C}-\mathrm{C}$ stretching and symmetric breathing vibrations of the aromatic carbon rings, respectively ${ }^{32}$. The variation in the intensity ratio of the D-peak to the G-peak $\left(\mathrm{I}_{\mathrm{D}} / \mathrm{I}_{\mathrm{G}}\right)$ and the position of the G-peak can be used to characterize the change in the carbon atomic structure of carbon materials. The increased $\mathrm{sp}^{2} / \mathrm{sp}^{3}$ bonding causes the G-peak position to shift upward and the $\mathrm{I}_{\mathrm{D}} / \mathrm{I}_{\mathrm{G}}$ ratio to increase ${ }^{32}$. The $\mathrm{G}$-peak position and the $\mathrm{I}_{\mathrm{D}} / \mathrm{I}_{\mathrm{G}}$ ratio in the Raman spectra of the carbon materials as a function of the deposition pressure are also shown in Fig. 3b. It should be noted that the variation in the $\mathrm{G}$-peak position and the $\mathrm{I}_{\mathrm{D}} / \mathrm{I}_{\mathrm{G}}$ ratio can be divided into two stages. One stage occurs at a relatively low deposition pressure ( $\leq 200$ mTorr), where the G-peak position and $\mathrm{I}_{\mathrm{D}} / \mathrm{I}_{\mathrm{G}}$ ratio exhibit low values of approximately $1522.4 \mathrm{~cm}^{-1}$ and 0.52 , respectively. The 
a

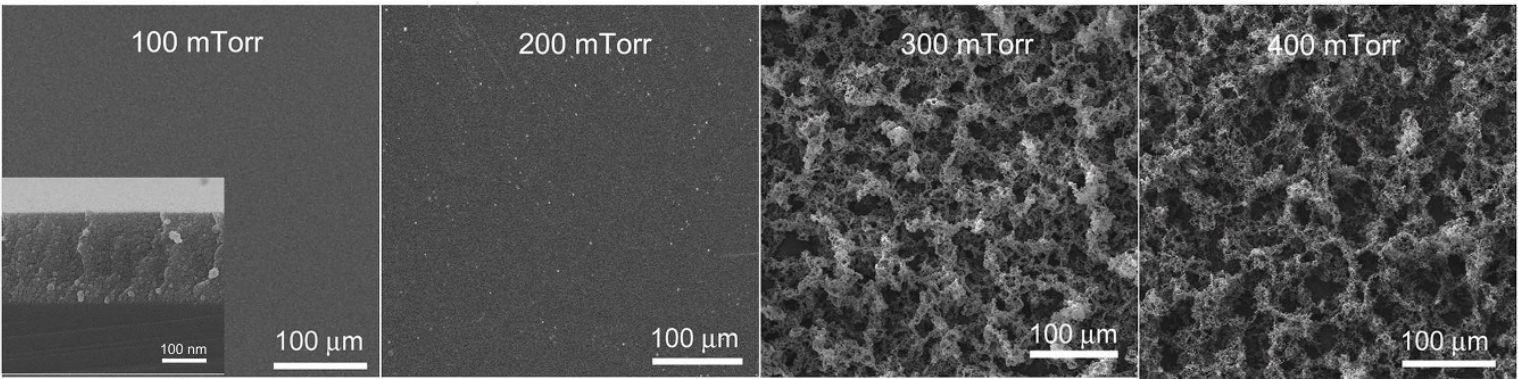

b
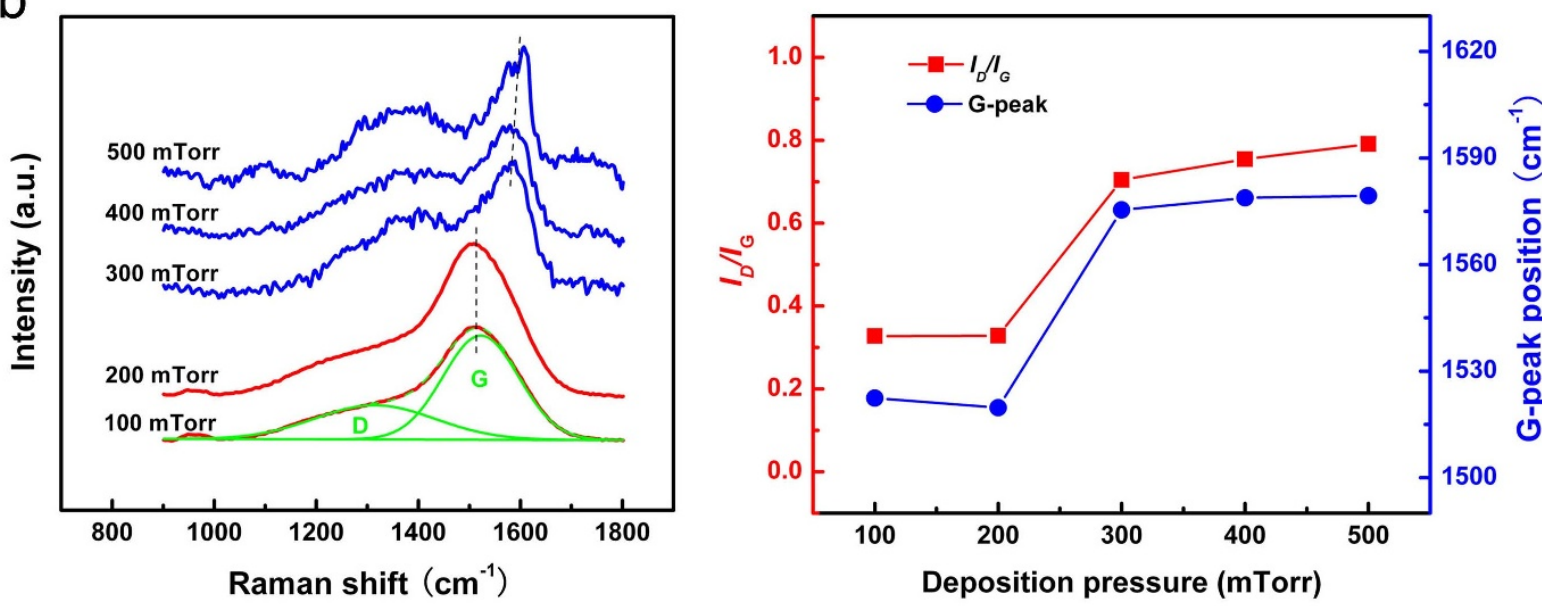

C

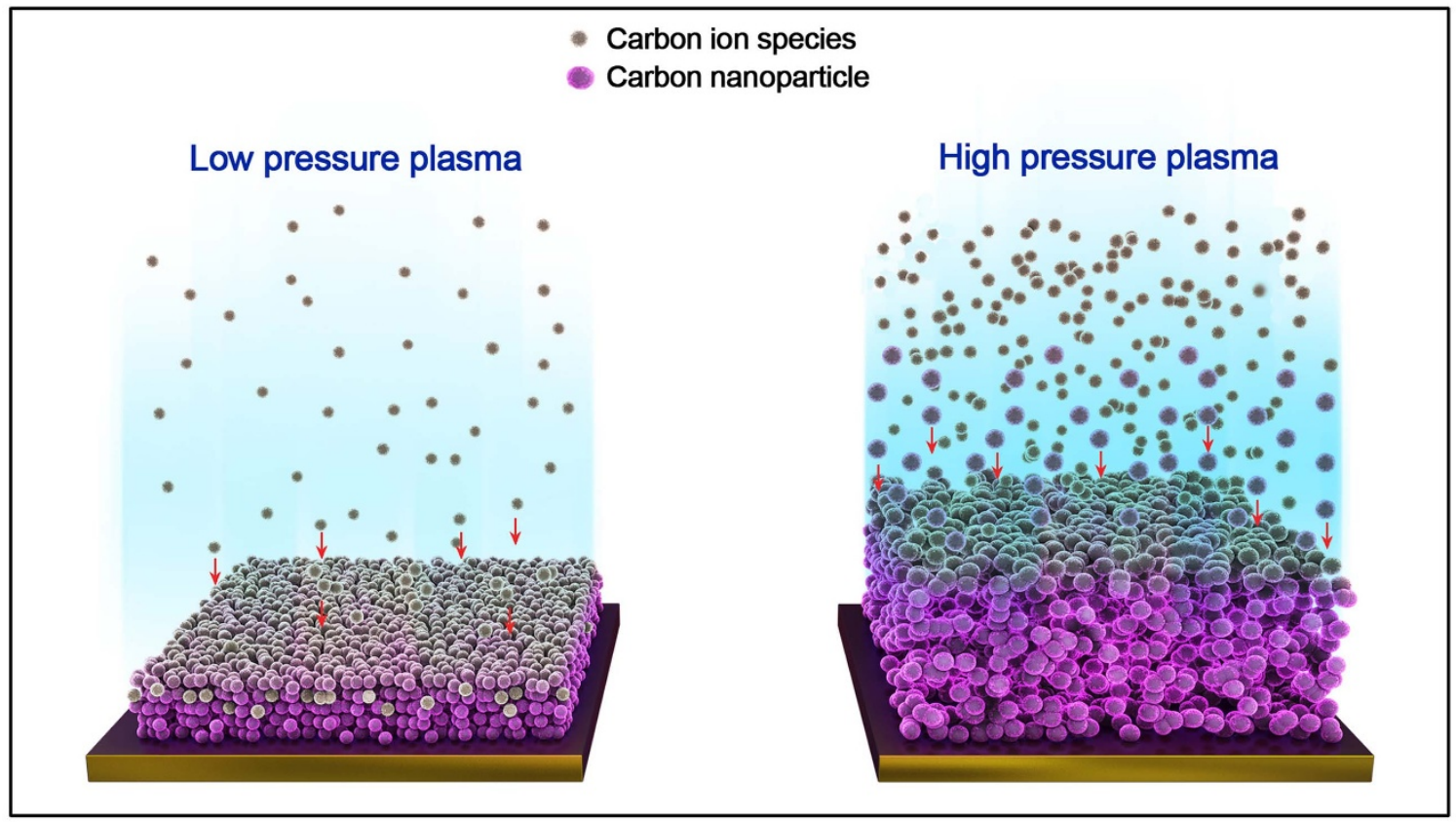

Figure $2 \mid$ Evolutions of the morphology, carbon bond structure and growth mechanism of as-deposited carbon materials as a function of the deposition pressure. (a) SEM images of carbon materials deposited at different pressures; (b) Raman spectra showing the corresponding $I_{D} / I_{G}$ ratios as well as the G-peak position of the carbon materials; (c) schematic of the as-deposited carbon materials grown at low and high working pressures.

other stage occurs at a relatively high deposition pressure ( $>200 \mathrm{mTorr}$ ), where the $\mathrm{G}$-peak position and $\mathrm{I}_{\mathrm{D}} / \mathrm{I}_{\mathrm{G}}$ ratio have large values of approximately $1575.4 \mathrm{~cm}^{-1}$ and 2.15 , respectively. This implies that the amorphous carbon coatings deposited at a low pressure contain relatively high contents of $\mathrm{sp}^{3}$ bonds, while the porous CNP network coatings acquired at a high pressure mainly contain $\mathrm{sp}^{2}$ bonds.
It is well known that the presence of fluorine in a carbon matrix reduces the surface energy remarkably and enhances the hydrophobicity of the resulting material ${ }^{33}$. Thus, $\mathrm{CF}_{4}$ was mixed with $\mathrm{C}_{2} \mathrm{H}_{2}$ for the deposition of fluorine-containing porous CNP network materials (an F-porous CNP network). Figure 3a shows SEM images of the porous CNP coatings deposited at a pressure of $500 \mathrm{mTorr}$ as a function of the $\mathrm{CF}_{4} / \mathrm{C}_{2} \mathrm{H}_{2}$ ratio in the gas mixtures of $\mathrm{CF}_{4}$ and 
a
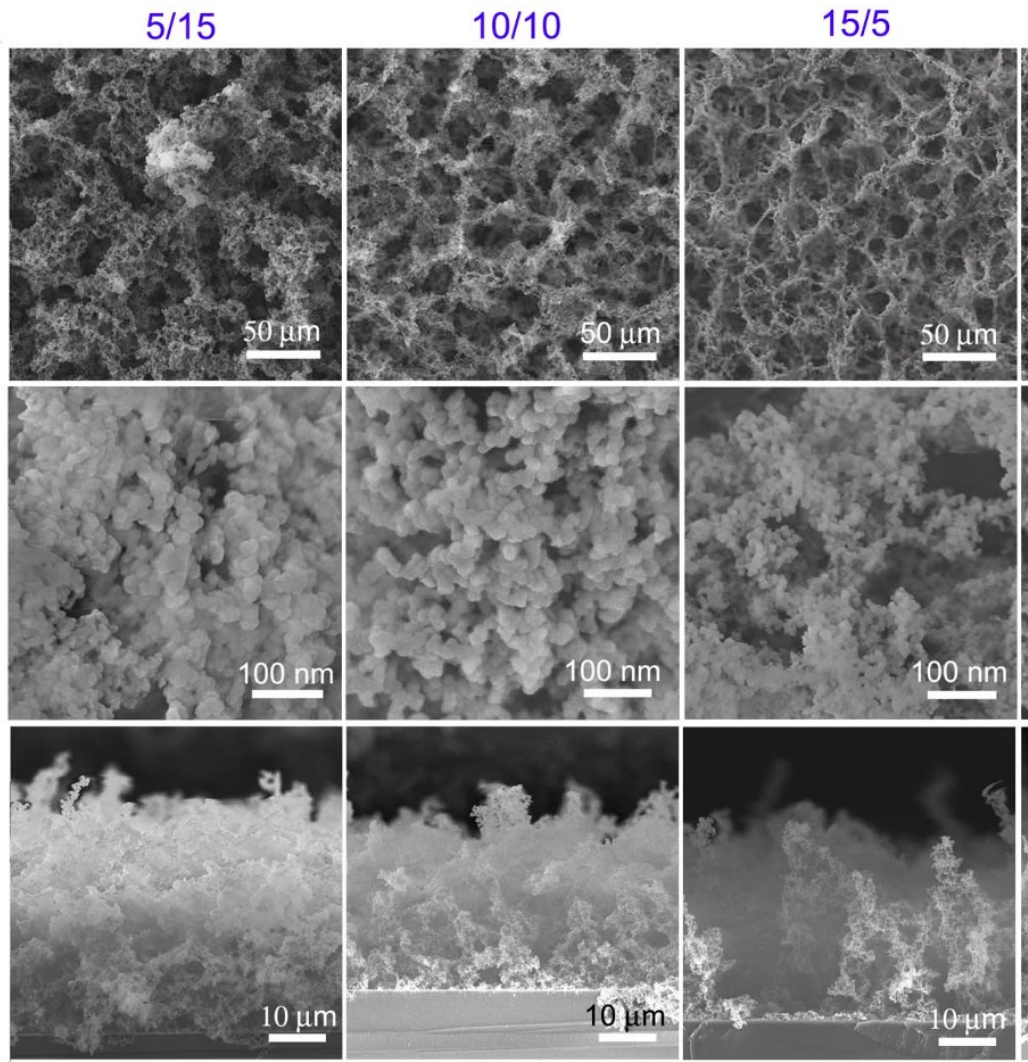

b

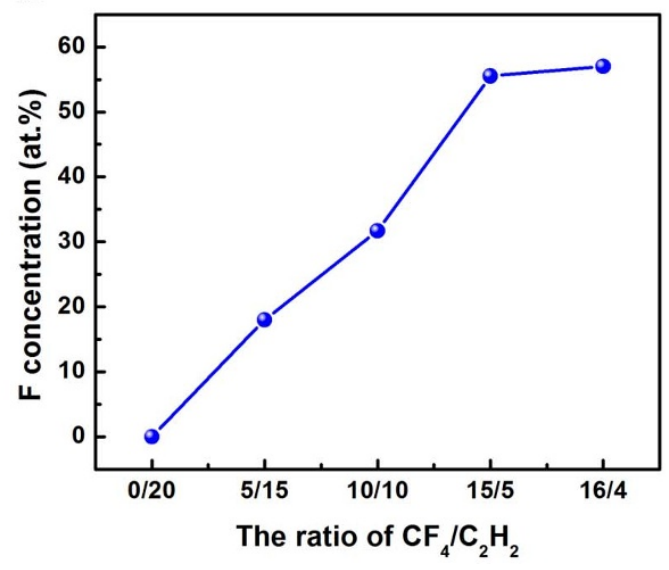

C

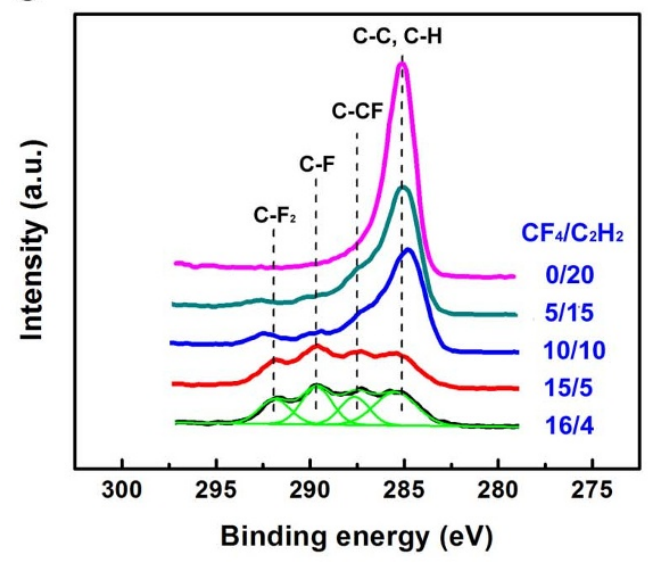

Figure 3 Morphology and chemical structure of fluorine-contained porous CNP materials. (a) SEM images of F-porous CNP network materials deposited at various $\mathrm{CF}_{4} / \mathrm{C}_{2} \mathrm{H}_{2}$ ratios at a deposition pressure of $500 \mathrm{mTorr}$; (b) fluorine concentration and (c) the C 1s XPS spectra as a function of the $\mathrm{CF}_{4} / \mathrm{C}_{2} \mathrm{H}_{2}$ ratio.

$\mathrm{C}_{2} \mathrm{H}_{2}$. It should be noted that the F-porous CNP network coatings exhibited porous structures similar to those of pure porous CNP network coatings. However, as the $\mathrm{CF}_{4}$ was incorporated, the porous CNP network coatings became more porous and the network and bridge structures became clearer. At the same time, the average size of the nanoparticles decreased from approximately $30 \mathrm{~nm}$ to $5 \mathrm{~nm}$ as the $\mathrm{CF}_{4} / \mathrm{C}_{2} \mathrm{H}_{2}$ ratio increased from $0 / 20$ to $16 / 4$. The cross-sectional images show that the as-deposited carbon materials became loose in terms of their microstructure and that the thickness of the carbon materials decreased as the $\mathrm{CF}_{4} / \mathrm{C}_{2} \mathrm{H}_{2}$ ratio increased. The BET measurement also indicates that the specific surface area of the F-porous CNP networks increases by about $50 \%$ compared to that of a pure porous CNP network (see Fig. S1). The introduction of $\mathrm{CF}_{4}$ reduced the carbon ion density of the plasma and thus prevented the growth of CNPs. The fluorine concentration of the as-deposited F-porous CNP network materials was determined by comparing the normalized area intensities of the $\mathrm{C} 1 \mathrm{~s}$ and $\mathrm{F} 1 \mathrm{~s}$ peaks as obtained from XPS measurements; the contribution of hydrogen was neglected because it could not be detected by XPS. Figure $3 \mathrm{~b}$ shows the fluorine concentration as a function of the $\mathrm{CF}_{4} / \mathrm{C}_{2} \mathrm{H}_{2}$ ratio $(0 / 20$ corresponding to only $\mathrm{C}_{2} \mathrm{H}_{2}$ ). It can be seen that the fluorine concentration increased monotonously from 0 to 57 at. \% as the ratio of $\mathrm{CF}_{4} /$ $\mathrm{C}_{2} \mathrm{H}_{2}$ increased from $0 / 20$ to $16 / 4$. Figure $3 \mathrm{c}$ presents the $\mathrm{C} 1 \mathrm{~s}$ XPS spectra of the $\mathrm{F}$-porous carbon materials deposited with various ratios of $\mathrm{CF}_{4} / \mathrm{C}_{2} \mathrm{H}_{2}$. The pure porous $\mathrm{CNP}$ network materials (0/ 20) reveal only a single peak around $285 \mathrm{eV}$; this was assigned to the $\mathrm{C}-\mathrm{C}$ or $\mathrm{C}-\mathrm{H}$ bond, representing the characteristic $\mathrm{C}$ 1s XPS spectrum of amorphous carbon, in agreement with the Raman spectra 
a

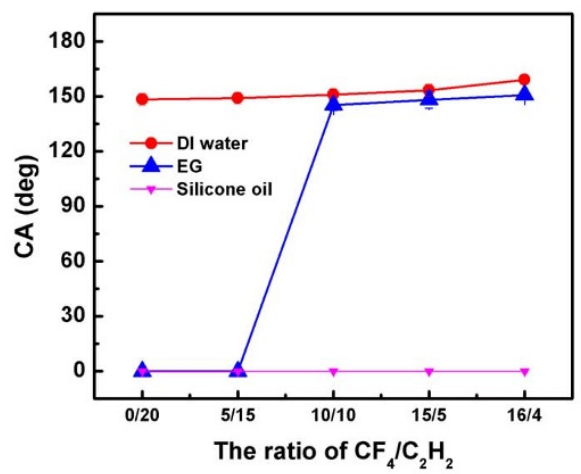

b

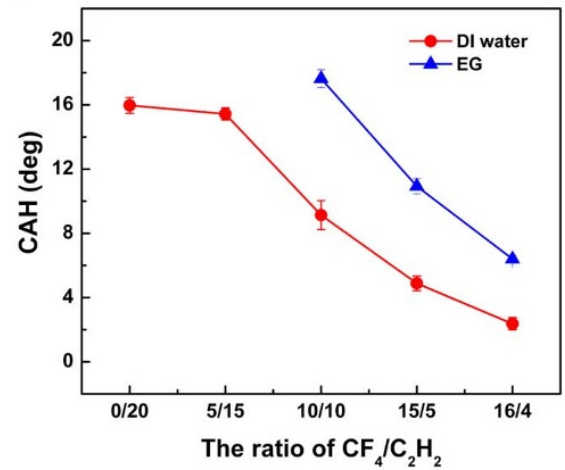

C
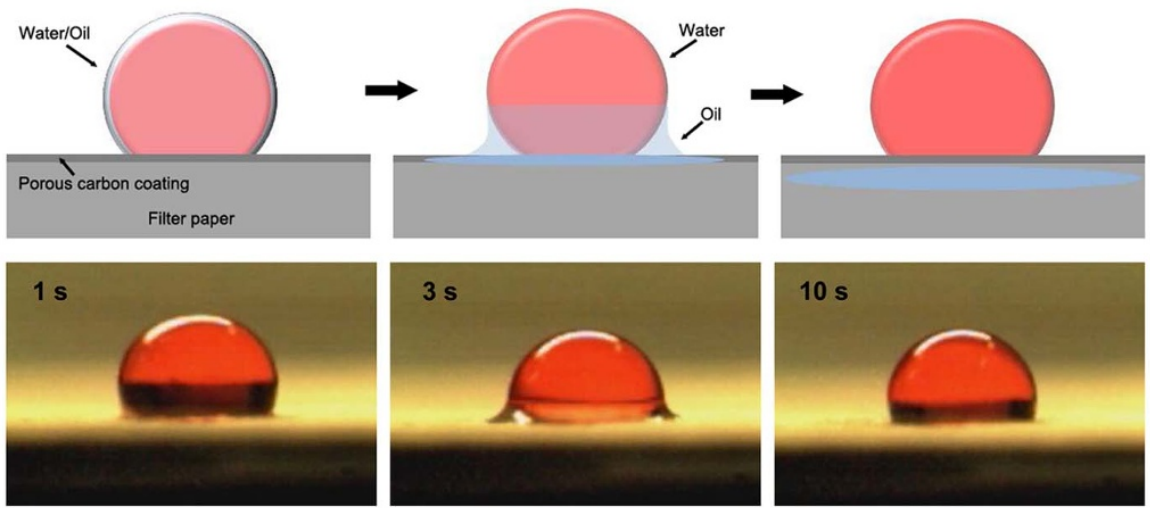

d

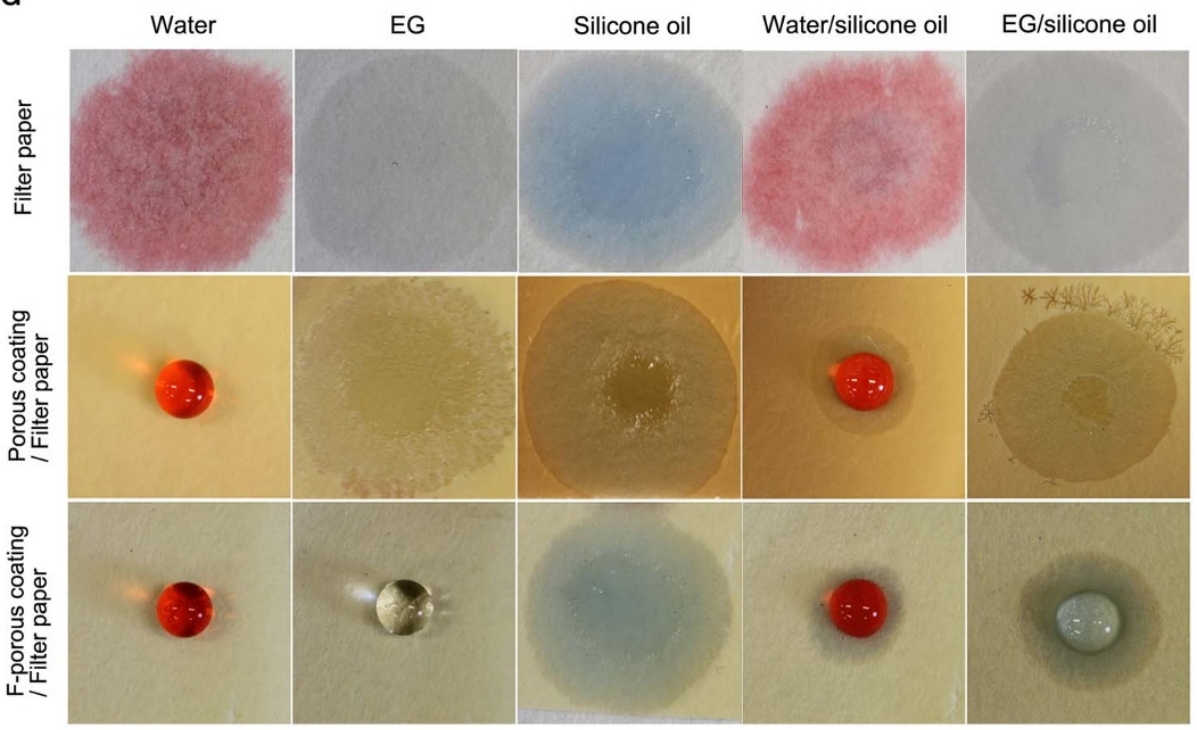

e

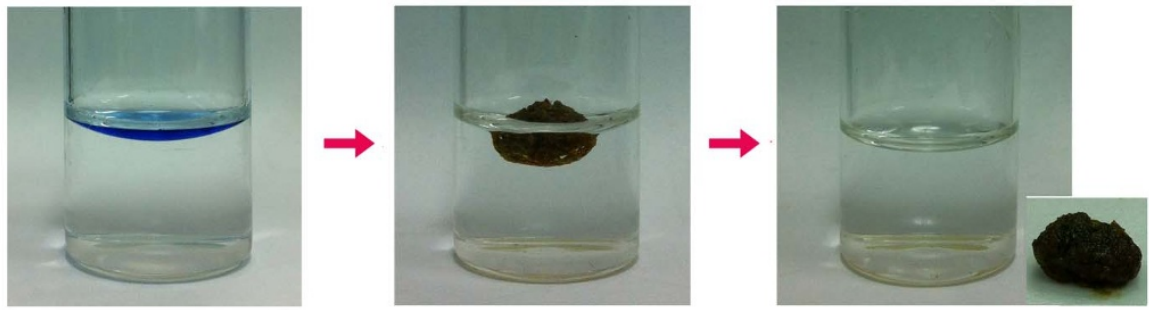

Figure $4 \mid$ Wetting behavior and oil absorption application. (a) static CAs and (b) CAHs of the as-deposited porous CNP network materials as a function of the $\mathrm{CF}_{4} / \mathrm{C}_{2} \mathrm{H}_{2}$ ratio in the precursor; (c) a schematic design of the water/oil separation process on the porous CNP network coating/filter paper (top), and photographs of the water/silicone oil mixture placed on the F-porous CNP network coating/filter paper (below); (d) the states of the water, E.G., silicone oil and their mixtures dropped on the surfaces of the filter paper and paper coated with porous carbon and F-porous carbon. The water droplet was dyed red, the silicone oil blue, and E.G. was clear; (e) a layer of silicone oil on the water can be removed by adding superhydrophobic porous CNP. 
analysis. With the increase in the $\mathrm{CF}_{4} / \mathrm{C}_{2} \mathrm{H}_{2}$ ratio, the relative intensity of the C-C peak decreased sharply and some new peaks began to appear at a high binding energy. For the F-porous CNP network materials deposited at relative high ratios of $\mathrm{CF}_{4} / \mathrm{C}_{2} \mathrm{H}_{2}(15 / 5$ and $16 / 4$ ), the $\mathrm{C} 1 \mathrm{~s}$ spectra can be deconvoluted into four components with binding energies of approximately $287.5,289.5$, and $292 \mathrm{eV}$, which correspond to the $\mathrm{C}-\mathrm{CF},-\mathrm{CF}$, and $-\mathrm{CF}_{2}$ bonding states, respectively. The relative intensity of the $\mathrm{C}-\mathrm{C}($ or $\mathrm{C}-\mathrm{H})$ bonds decreased sharply in the spectra due to the significant decrease in the $\mathrm{C}_{2} \mathrm{H}_{2}$ fraction. The $\mathrm{CF}_{4}$ molecules were decomposed easily into various radicals in the plasma, and these fluorine-containing radicals subsequently saturated the unsaturated $\mathrm{C}$ bonds and replaced $\mathrm{C}-\mathrm{H}$ with $\mathrm{CF}_{\mathrm{x}}$ species because $\mathrm{C}-\mathrm{H}$ bonds are weaker than $\mathrm{C}-\mathrm{F}$ bonds ${ }^{34}$. As a result, the dominant bonding states of the as-deposited porous CNP network materials are $\mathrm{CF}_{\mathrm{x}}$ groups at high $\mathrm{CF}_{4} / \mathrm{C}_{2} \mathrm{H}_{2}$ ratios. The formation of $\mathrm{CF}_{\mathrm{x}}$ groups reduced the dispersive component, polarizability, and surface energy of the F-porous materials and thus improved their hydrophobicity ${ }^{33}$.

The static CAs and corresponding CAHs measured for the Fporous $\mathrm{CNP}$ network materials deposited with various $\mathrm{CF}_{4} / \mathrm{C}_{2} \mathrm{H}_{2}$ ratios are shown in Figs. $4 \mathrm{a}$ and b. It should be noted that as the $\mathrm{CF}_{4} / \mathrm{C}_{2} \mathrm{H}_{2}$ ratio in the gas mixture increased, the static water $\mathrm{CA}$ of the F-porous CNP network coatings increased, whereas the $\mathrm{CAH}$ dropped. When the $\mathrm{CF}_{4} / \mathrm{C}_{2} \mathrm{H}_{2}$ ratio was $16 / 4$, the porous $\mathrm{CNP}$ coatings presented superhydrophobicity with a high CA of approximately $160^{\circ}$ and a low CAH of approximately $2^{\circ}$. We also investigated the wetting behavior of the F-porous CNP network coatings against different liquids of ethylene glycol (E.G.) and silicone oil, whose surface energies are 47.7 and $21.2 \mathrm{~mJ} / \mathrm{m}^{2}$ at $20^{\circ} \mathrm{C}$, respectively (the surface energy of DI water is $72.8 \mathrm{~mJ} / \mathrm{m}^{2}$ ). For E.G., the pure porous CNP network coatings as well as the network deposited with a low $\mathrm{CF}_{4} / \mathrm{C}_{2} \mathrm{H}_{2}$ ratio of $5 / 15$ revealed complete wetting behavior, with a CA of approximately $0^{\circ}$; in particular, E.G. was absorbed by the porous carbon, but at a high $\mathrm{CF}_{4} / \mathrm{C}_{2} \mathrm{H}_{2}$ ratio of $16 / 4$, the porous CNP network coatings showed hydrophobicity, with a CA of approximately $145^{\circ}$. As the $\mathrm{CF}_{4} / \mathrm{C}_{2} \mathrm{H}_{2}$ ratio was increased further, the CA increased monotonously with the decrease in the $\mathrm{CAH}$. When the $\mathrm{CF}_{4} / \mathrm{C}_{2} \mathrm{H}_{2}$ ratio reached $16 / 4$, the $\mathrm{CA}$ increased to $150^{\circ}$ and the $\mathrm{CAH}$ dropped to $7^{\circ}$, indicating superhydrophobicity. This extreme wetting contrast of E.G. on the F-porous CNP networks can be explained by the tunability of the coating materials with the $\mathrm{CF}_{4} / \mathrm{C}_{2} \mathrm{H}_{2}$ ratio (See Fig. S3) and by the surface roughness of the nanoscale porous structures. However, for the silicone oil, all samples with F-porous CNP coatings were completely wetted with oil due to the low surface energy of both liquids.

The wettability measurements showed that the pure porous CNP network deposited with only a hydrocarbon precursor can repel water very well and absorb low-surface-energy liquids (both E.G. and silicone oil). However, the F-porous CNP network with a high content of added fluorine can repel both water and E.G. but not silicone oil, which has the lowest surface energy. This means that we can tune the wettability or absorbability of the porous CNP networks by controlling the amount of $\mathrm{CF}_{4}$ that is incorporated. Thus, porous CNP networks can be used to separate mixtures of liquids with different surface tensions, such as water/silicone oil and E.G./ silicone oil. To test their liquid separation behavior, the pure porous CNP and F-porous CNP networks were coated onto filter paper. It was expected that when the water-silicone oil mixture was dropped onto the porous CNP network coating/filter paper system, the wetting phase of the silicone oil would be absorbed by the porous CNP network as the non-wetting phase of DI water was repelled and retained on the surface, as shown in Fig. $4 c^{35}$. Using a high-speed camera to record the behavior, a DI water droplet (dyed red) was bounced on the porous CNP network material without penetration (see video S1), while silicone oil (dyed in light blue) was quickly absorbed by the porous network/filter paper (see video S2). As the water/silicone oil mixture was placed on filter paper coated with a porous CNP network, the silicone oil surrounding the outer surface of the mixture droplet was quickly absorbed by the porous CNP, forming an oil meniscus (see movie S3) and leaving only a water droplet on the surface.

Figure $4 \mathrm{~d}$ shows the wetting states of the water, E.G., silicone oil and mixed droplets of these materials on the surfaces of the asreceived filter paper and the porous CNP and F-porous CNP networks coated onto filter paper. When each droplet was gently placed on each surface, $1 \mathrm{~min}$ was allowed to elapse before the optical images were taken. It is clear that all of the liquid droplets, i.e., those of water, E.G., silicone oil and their mixtures, were completely absorbed on the bare filter paper due to its hydrophilic and oleophilic nature, as shown in the first row of Fig. 4d. For the porous CNP coating/filter paper sample shown in the second row, the DI water droplet was repelled by the porous CNP network coating without penetration, while E.G. and silicone oil were completely absorbed. When the water/silicone oil mixture was dropped, only water remained on the surface, indicating the separation of the water from the silicone oil, whereas the E.G./silicone oil mixture was completely absorbed. For the F-porous CNP coated onto filter paper, DI water and E.G. were repelled while silicone oil was completely absorbed, as shown in the images in the last row. Accordingly, the F-porous CNP coating/filter paper system absorbed only silicone oil from the water/ silicone oil and E.G./silicone oil mixtures. This implies that porous CNP networks with a tunable fluorine fraction can be used to separate mixtures of liquids with different surface energies. The porous carbon materials collected from the Si wafers (as shown in the optical image of Fig. 1e) could also be used directly as absorbents for oil or organic solvents, such as hexane and toluene, as demonstrated in Fig. 4e. The porous CNP material was dropped into the water/silicone oil mixture and the water/hexane mixture, where only silicone oil (Fig. 4e and video S4(a)) and hexane (as shown in video S4(b)) were quickly absorbed, respectively. Furthermore, the selective wettability and absorbability of oil were maintained when the porous CNP material was immersed in water (as shown in Fig. S4). Even if the oil/water mixture was stirred, the small oil droplets under the water could also be absorbed easily by the porous CNP materials (shown in Fig. S5).

\section{Discussion}

The formation of the porous CNP materials was significantly correlated with the deposition pressure. Porous CNP structures were only observed above $300 \mathrm{mTorr}$, whereas the relatively smooth surfaces were observed in materials deposited at $\leq 200$ mTorr, as shown in Fig. 2a. It appears that there is a critical pressure $\left(P_{c}\right)$ between $200 \mathrm{mTorr}$ and $300 \mathrm{mTorr}$ at which a morphological transition in carbon microstructures occurs, as indicated by the present experiments. It is well known that the deposition pressure has a strong effect on the ion energy. In frequency discharges, the ion energy $E$ is related to the discharge voltage $V$ and deposition pressure $P$ via $E \propto V / \sqrt{P}$, and this is significantly correlated with the growth mode of carbon materials ${ }^{36}$. At a relatively low deposition pressure (lower than $P_{c}$ ), carbon ion species have sufficient energy to penetrate the growth subsurface, resulting in an increase in the local density of the material as well as the promotion of $\mathrm{sp}^{3}-\mathrm{C}$ bond formation due to high energy accumulation, similar to that observed during the growth of the amorphous carbon film (as shown in Fig. 2c) ${ }^{32}$. However, at a high deposition pressure (higher than $P_{c}$ ), more collisions take place among the gas molecules, ions, and electrons in the plasma, which thus increase the plasma's radical density and reduce the energy of the carbon ion species. As a result, CNPs form in the dense plasma and grow as the density of the carbon radicals increases (carbon ion species), like other nanoparticles deposited via CVD processes $^{37-39}$. Because the energy was too low for the CNPs to penetrate the growing subsurface, the CNPs simply adhered to the 
growing top surface and remained in the lowest energy state of $\mathrm{sp}^{2}$ bonding (graphite-like bonding) ${ }^{32}$, forming a porous network as nano-building blocks with the continuous aggregation of nanoparticles on the porous surface.

It is evident that the superhydrophobicity of the porous CNPs materials was mainly due to the fractal-like roughness surface consisting of CNPs, which allowed air to be trapped between the particle networks and which prevented the penetration of water into the porous network ${ }^{4,8}$. In addition, enhanced superhydrophobicity of the porous carbon structures was achieved by reducing the surface energy by increasing the fluorine content, as indicated by the increase in the water contact angle of the structures formed on the flat and smooth surfaces at a low pressure (shown in Fig. S3). The equilibrium state of a water droplet on a surface with a porosity fraction $f_{p}=(1-\phi)$ (where $\phi$ is the solid fraction of surface area at the top of the asperities) can be considered a Cassie-Baxter state, with the apparent contact line of the droplet represented by $\cos \theta_{a p p}^{W}=$ $\phi\left(\cos \theta_{e}+1\right)-1$, where $\theta_{e}$ and $\theta_{a p p}^{W}$ are the equilibrium and apparent $\mathrm{CA}$, respectively ${ }^{40}$. This suggests that as the porosity and equilibrium CA (as measured on a flat surface) increase, the hydrophobicity increases. The incorporation of $\mathrm{CF}_{4}$ not only increased the equilibrium $\mathrm{CA}$ on the flat carbon coatings via the reduced surface energy but also increased the porosity of the porous carbon. Therefore, we can tune the wettability of porous CNP networks by controlling the amount of $\mathrm{CF}_{4}$ that is incorporated.

The sharp change of the wettability by tuning the surface energy of the F-porous carbon may come from the change of the wetting state. Bico et al. ${ }^{41}$ studied the critical condition necessary for a structured surface to absorb a liquid, revealing that such a condition could be expressed as a critical contact angle, as follows:

$$
\theta_{c}=\cos ^{-1}\left(\frac{1-\phi}{r-\phi}\right)
$$

Here, $\theta_{c}, \phi$ and $r$ are the critical contact angle, roughness fraction and roughness as defined as the ratio of the real surface area to the projected area. In this equation, the equilibrium contact angle of the liquid should be less than the critical contact angle shown above for the liquid to be absorbed into the specimen. Because the roughness, $r$, of a porous type of media is considered to be almost infinite, $\infty$, the critical contact angle of a porous type of media is known to be $90^{\circ}$ theoretically. When such absorption occurs, the contact angle should be $0^{\circ}$ because the liquid droplet is fully absorbed into the porous media. Moreover, if the equilibrium contact angle is higher than $90^{\circ}$, a liquid droplet would be in a Cassie-Baxter state, resulting in generally a higher contact angle than $150^{\circ}$ and lower contact angle hysteresis of less than $20^{\circ}$. However, recent studies have revealed that a Cassie-Baxter state could occur even on a hydrophilic surface with contact angles lower than $90^{\circ}$ when the surface roughness has a structure that easily traps air or that has a sharp geometry ${ }^{42}$. Experimental data show that a Cassie-Baxter state arises when the equilibrium contact angle exceeds approximately $50^{\circ}$, as shown in Figs. $4 \mathrm{a}-\mathrm{b}$ and Fig. S3. The contact angles of water and silicone oil are always over $50^{\circ}$ and $0^{\circ}$, respectively. Hence, the contact angles of water and silicone oil are dominant under a Cassie-Baxter state and an absorption state, respectively. However, for E.G., the equilibrium contact angle is varied from $0^{\circ}$ to $80^{\circ}$ by tuning the ratio of $\mathrm{CF}_{4} / \mathrm{C}_{2} \mathrm{H}_{2}$. Therefore, only E.G. undergoes a transition from the absorption state to the Cassie-Baxter state, resulting in a severe increase in the CA from $0^{\circ}$ to about $150^{\circ}$. Thus, porous CNP networks with tunable porosity and surface energy can be used to separate mixtures of liquids with different surface tensions, such as water/silicone oil and E.G./silicone oil mixtures.

Our porous CNP materials could easily be synthesized on various materials, such as metals, polymers or paper, at a high yield by hydrocarbon gas glow discharge. Furthermore, varying the fraction of $\mathrm{CF}_{4}$ gas in the hydrocarbon precursor allows the tuning of the surface energy of the porous CNP networks and allows for the selective repellency and absorption of liquids with different surface tensions, making it possible to separate mixtures of liquids. These selectively repellent or absorbent porous CNP networks can be used as a benign material in various applications, including water filtration and liquid separation or oil-spill (or organics) cleanup.

\section{Methods}

Deposition of carbon materials. A series of carbon materials were deposited by glow discharge on silicon wafers (100) at a thickness of $525 \pm 15 \mu \mathrm{m}$. Filter paper was also used as a substrate to characterize the absorbability of the as-deposited carbon materials. The Si wafers were cleaned in an ultrasonic bath in acetone and ethanol and dried under a nitrogen flow before being placed in a vacuum chamber. The chamber was evacuated to a base pressure of approximately $2 \times 10^{-5}$ Torr. $\mathrm{C}_{2} \mathrm{H}_{2}$ was introduced into the chamber at a flux rate of $20 \mathrm{sccm}$ as the carbon precursor. The deposition temperature of the samples placed on the cathode was held at room temperature by water cooling, and the work pressure was varied from 100 to 500 mTorr. In addition, gas mixtures with various $\mathrm{CF}_{4} / \mathrm{C}_{2} \mathrm{H}_{2}$ ratios $(5 / 15,10 / 10,15 / 5$ and 16/4) were used as the precursors to prepare fluorine-contained carbon materials at two different deposition pressures of 100 and $500 \mathrm{mTorr}$, corresponding to flat and porous carbon in terms of their configuration. The discharge was sustained at a radio frequency $(\mathrm{RF})$ and power of $13.56 \mathrm{MHz}$ and $600 \mathrm{~W}$, respectively, for a total process period of $3 \mathrm{~min}$.

Characterization methods. A scanning electron microscope (SEM, Nova 600, FEI) operated at an accelerating voltage of $5 \mathrm{keV}$ was used to observe the porous CNP networks. A transmission electron microscope (TEM, Tecnai G2, FEI) equipped with a cold-field emission gun and operated at $200 \mathrm{keV}$ provided microscopic information regarding the porous CNP networks. TEM specimens were prepared by dispersing the porous CNPs in isopropanol and then dropping them onto a holey $\mathrm{Cu}$ grid. The surface area and pore distribution of the carbon materials were determined by the BET method using an ASAP-2020 V3.00 Q-physisorption system using $\mathrm{N}_{2}$ as the adsorbate at $77 \mathrm{~K}$. Before measuring the surface area, the sample was outgassed at $120^{\circ} \mathrm{C}$ for 10 hours to remove the physisorbed water molecules. The bonding nature of the carbon materials was characterized using Raman spectroscopy with incident light from a Xe $\mathrm{e}^{+}$laser at a wavelength of $532 \mathrm{~nm}$. The Raman scattering range spanned from 700 to $2000 \mathrm{~cm}^{-1}$. Compositional analyses were performed using Xray photoelectron spectroscopy (XPS) to investigate the chemical change in the porous $\mathrm{CNP}$ networks with an increase in the degree of $\mathrm{CF}_{4}$ incorporation. $\mathrm{An} \mathrm{Al} \mathrm{K \alpha}$ $(1486.6 \mathrm{eV}) \mathrm{X}$-ray source was used as the excitation source for XPS, and the X-ray source anode was maintained at $250 \mathrm{~W}, 10 \mathrm{kV}$, and $27 \mathrm{~mA}$ with a beam spot size of $400 \mu \mathrm{m} \times 400 \mu \mathrm{m}$. The XPS peak position was calibrated using the $\mathrm{C} 1 \mathrm{~s}$ peak at $284.6 \mathrm{eV}$.

The wettability of the porous CNP network coated onto the Si wafers was characterized by measuring the $\mathrm{CA}$ and $\mathrm{CAH}$ of deionized (DI) water droplets. For these measurements, droplets of approximately $5 \mu \mathrm{L}$ were gently deposited on the substrates using a microsyringe. The $\mathrm{CAH}$ was calculated by the difference between the measured advancing and receding CAs, where the advancing CA was measured by adding a DI water sessile drop $(\sim 5 \mu \mathrm{L})$ and the receding CA was determined by the removal of water from the DI water sessile drop. All measurements were made using a contact angle goniometer (ramé-hart) in ambient air at room temperature with a relative humidity of $20 \sim 35 \%$. The reported CA values were determined by averaging the measurements from five different spots on each sample. Liquid separation was performed using three liquid mixtures, water/silicone oil, E.G./silicone oil, and water/ hexane, on filter paper coated with and without porous CNPs. A high-speed camera (Motionpro X4, Redlake) was used to record the absorbing behaviour of the water/ silicone oil mixture on the filter paper.

1. Shirtcliffe, N. J., McHale, G., Atherton, S. \& Newton, M. I. An introduction to superhydrophobicity. Adv. Colloid Interf. Sci. 161, 124-138 (2010).

2. Guo, Z., Liu, W. \& Su, B. L. Superhydrophobic surface: from natural to biomimetic to functional. J. Colloid Interf. Sci. 353, 335-355 (2011).

3. Li, X. M., Reinhoudt, D. \& Crego-Calama, M. What do we need for a superhydrophobic surface? A review on the recent progress in the preparation of superhydrophobic surface. Chem. Soc. Rev. 36, 1350-1368 (2007).

4. Deng, X., Mammen, L., Butt, H. J. \& Vollmer, D. Candle soot as a template for a transparent robust superamphiphobic coating. Science 335, 67-70 (2012).

5. Banerjee, D., Mukherjee, S. \& Chattopadhyay, K. K. Controlling the surface topology and hence the hydrophobicity of amorphous carbon thin films. Carbon 48, 1025-1031 (2010).

6. Zhou, Y. et al. Control over the wettability of amorphous carbon films in a large range from hydrophilicity to super-hydrophobicity. Appl. Surf. Sci. 253, 2690-2694 (2006).

7. Yan, A., Xiao, X., Külaots, I., Sheldon, B. W. \& Hurt, R. H. Controlling water contact angle on carbon surfaces from $5^{\circ}$ to $167^{\circ}$. Carbon 44, 3113-3148 (2006)

8. Singh, E. et al. Superhydrophobic Graphene Foams. Small. 9, 75-80 (2013).

9. Dong, X. et al. Superhydrophobic and superoleophilic hybrid foam of graphene and carbon nanotuble for selective removal of oils or organic solvents from the surface of water. Chem. Commun. 48, 10660-10662 (2010). 
10. Lau, K. S. K. et al. Superhydrophobic carbon nanotube forests. Nano Lett. 3, 1701-1705 (2003).

11. Ko, T. J. et al. Water condensation behavior on the surface of a network of superhydrophobic carbon fibers with high-aspect-ratio nanostructures. Carbon 50, 5085-5092 (2012).

12. Feng, L. et al. Superhydrophobicity of nanostructured carbon films in wide range of pH values. Angew. Chem. Int. Ed. 115, 4349-4352 (2003).

13. Wang, L. et al. Super-hydrophobic ordered mesoporous carbon monolith. Carbon 44, 1336-1339 (2006).

14. Hu, Y.-S. et al. Synthesis of hierarchically porous carbon monoliths with highly ordered microstructure and their application in rechargeable lithium batteries with high-rate capability. Adv. Funct. Mater. 17, 1873-1878 (2007).

15. Zhu, Y. et al. Carbon-based supercapacitors produced by activation of graphene. Science 332, 1537-1541 (2011).

16. Wang, D. W., Li, F., Liu, M., Lu, G. Q. \& Cheng, H. M. 3D a periodic hierarchical porous graphitic carbon material for high-rate electrochemical capacitive energy storage. Angew. Chem. 120, 379-382 (2008).

17. Wang, H., Gao, Q. \& Hu, J. High hydrogen storage capacity of porous carbons prepared by using activated carbon. J. Am. Chem. Soc. 131, 7016-7022 (2009).

18. Yu, J. S., Kang, S., Yoon, S. B. \& Chai, G. Fabrication of ordered uniform porous carbon networks and their application to a catalyst supporter. J. Am. Chem. Soc. 124, 9382-9383 (2002).

19. Nguyen, D. D., Tai, N. H., Lee, S. B. \& Kuo, W. S. Superhydrophobic and superoleophilic properties of graphene-based sponges fabricated using a facile dip coating method. Energy Environ. Sci. 5, 7908-7912 (2012).

20. Mecklenburg, M. et al. Aerographite: ultra lightweight, flexible nanowall, carbon microtube material with outstanding mechanical performance. Adv. Mater. 24, 3486-2490 (2012).

21. Mauter, M. S. \& Elimelech, M. Environmental applications of carbon-based nanomaterials. Environ. Sci. Techol. 42, 5843-5859 (2008)

22. Lee, J., Kim, J. \& Hyeon, T. Recent progress in the synthesis of porous carbon materials. Adv. Mater. 18, 2073-2094 (2006).

23. Banerjee, D., Sen, D. \& Chattopadhyay, K. K. Simple chemical synthesis of porous carbon spheres and its improved field emission by water vapor adsorption. Microporous Mesoporous Mater. 171, 201-207 (2013).

24. Chuenchom, L., Kraehnert, R. \& Smarsly, B. M. Recent progress in soft-templating of porous carbon materials. Soft Matter 8, 10801-10812 (2012).

25. Gui, X. et al. Carbon nanotube sponges. Adv. Mater. 22, 617-621 (2010).

26. Fan, S. Self-oriented regular arrays of carbon nanotubes and their field emission properties. Science 283, 512-514 (1999).

27. Wu, Y., Qiao, P., Chong, T. \& Shen, Z. Carbon nanowalls grown by microwave plasma enhanced chemical vapor deposition. Adv. Mater. 14, 64-67 (2002).

28. Zhang, G., Jiang, X. \& Wang, E. Tubular graphite cones. Science 300, 472-474 (2003).

29. Lin, H., Gerbec, J. A., Sushchikh, M. \& McFarland, E. W. Synthesis of amorphous silicon carbide nanoparticles in a low temperature low pressure plasma reactor. Nanotechnology 19, 325601.1-325601.8 (2008).

30. Kim, Y. A., Muramatsu, H., Hayashi, T. \& Endo, M. Catalytic metal-free formation of multi-walled carbon nanotubes in atmospheric arc discharge. Carbon 50, 4588-4595 (2012).

31. Casiraghi, C., Ferrari, A. C. \& Robertson, J. Raman spectroscopy of hydrogenated amorphous carbons. Phys. Rev. B 72, 085401.1-085401.14 (2005).

32. Robertson, J. Diamond-like amorphous carbon. Mater. Sci. Eng., R 37, 129-281 (2002).
33. Yu, G. O., Tay, B. K., Sun, Z. \& Pan, L. K. Properties of fluorinated amorphous diamond like carbon films by PECVD. Appl. Surf. Sci. 219, 228-237 (2003).

34. Hikosaka, Y., Toyoda, H. \& Sugai, H. Spatial distribution and surface loss of $\mathrm{CF}_{3}$ and $\mathrm{CF}_{2}$ radicals in a $\mathrm{CF}_{4}$ etching plasma. Jpn. J. Appl. Phys. 32, L353-L356 (1993).

35. Kota, A. K., Kwon, G., Choi, W., Mabry, J. M. \& Tuteja, A. Hygro-responsive membranes for effective oil-water separation. Nat. Commun. 3, 1025 (2012).

36. Catherine, Y. \& Couderc, P. Electrical characteristics and growth kinetics in discharges used for plasma deposition of amorphous carbon. Thin Solid Films 144, 265-280 (1986).

37. Shen, Z., Kim, T., Kortshagen, U., McMurry, P. H. \& Campbell, S. A. Formation of highly uniform silicon nanoparticles in high density silane plasmas. J. Appl. Phys. 94, 2277-2283 (2003).

38. Hong, Y. C., Shin, D. H. \& Uhm, H. S. Production of vanadium nitride nanopowders from gas-phase $\mathrm{VOCl}_{3}$ by making use of microwave plasma torch. Mater. Chem. Phys. 101, 35-40 (2007).

39. Adachi, M., Tsukui, S. \& Okuyama, K. Nanoparticle formation mechanism in CVD reactor with ionization of source vapor. J. Nanopart. Res. 5, 31-37 (2003).

40. Cassie, A. B. D. \& Baxter, S. Wettability of porous surfaces. Trans. Faraday Soc. 40, 546-551 (1944)

41. Bico, J., Tordeux, C. \& Quéré, D. Rough wetting. Europhys. Lett. 55, 214-220 (2001).

42. Liu, J. L., Feng, X. Q., Wang, G. \& Yu, S. W. Mechanisms of superhydrophobicity on hydrophilic substrates. J. Phys.: Condens. Matter 19, 356002 (2007).

\section{Acknowledgements}

This work was financially supported in part by TES Co. Ltd. under the 'Advanced Manufacturing Technology Research Center' Program of the MKE of Korea (KRL) and the Eco-innovation program (Environmental Research Laboratory) (414-111-011 MWM). It was also supported in part by a grant from the Global Excellent Technology Innovation R\&D Program funded by the MKE (MWM, HYK), by National Research Foundation (grant no. 2013034978, HYK) and by a KIST internal project. The authors would like to thank $\mathrm{H}$. A. Stone and K.-S. Kim for the useful discussions.

\section{Author contributions}

M.W.M. and W.D. planned the study. W.D. performed materials deposition and some of the characterization steps. S.J.K., W.K.S. and S.H.K. carried out the wetting-related experiment, TEM analysis and BET measurement, respectively. K.R.L. and H.Y.K. helped to analyze the experimental data and results. W.D., M.W.M. and S.J.K. wrote the manuscript. K.R.L. and H.Y.K. discussed and commented on the manuscript. All authors provided feedback.

\section{Additional information}

Supplementary information accompanies this paper at http://www.nature.com/ scientificreports

Competing financial interests: The authors declare no competing financial interests.

How to cite this article: Dai, W. et al. Porous Carbon Nanoparticle Networks with Tunable Absorbability. Sci. Rep. 3, 2524; DOI:10.1038/srep02524 (2013).

\footnotetext{
(c) (i) (2) (2) This work is licensed under a Creative Commons Attribution-

Co No SA Nommercial-ShareAlike 3.0 Unported license. To view a copy of this license, visit http://creativecommons.org/licenses/by-nc-sa/3.0
} 
SUBJECT AREAS: NANOPARTICLES

SYNTHESIS AND PROCESSING

\section{SCIENTIFIC REPORTS:}

$3: 2524$

DOI: $10.1038 /$ srep02524

(2013)

Published:

28 August 2013

Updated:

20 November 2013
CORRIGENDUM: Porous Carbon Nanoparticle Networks with Tunable Absorbability

Wei Dai, Seong Jin Kim, Won-Kyung Seong, Sang Hoon Kim, Kwang-Ryeol Lee, Ho-Young Kim \& Myoung-Woon Moon

The original version of this Article contained a typographical error in the spelling of the author Won-Kyung Seong which was incorrectly given as Won-Kyeong Seong. This has been corrected in the HTML and PDF versions of the Article. 